\title{
Arterial tortuosity syndrome causing recurrent transient ischemic attacks in young adult: a case report
}

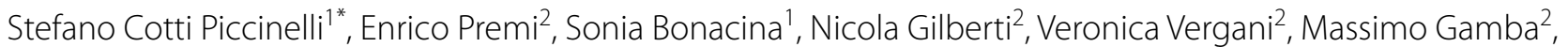
Raffaella Spezi ${ }^{2}$, Ilenia Delrio ${ }^{2}$, Michaël Bernier ${ }^{3}$, Angelo Costa², Marco Ravanelli ${ }^{4}$, Marina Colombi ${ }^{5}$, Roberto Gasparotti ${ }^{6}$, Alessandro Padovani ${ }^{1}$ and Mauro Magoni ${ }^{2}$

\begin{abstract}
Background: Arterial Tortuosity Syndrome (ATS) is a rare autosomal recessive disorder characterized by elongated and tortuous arteries. Although ATS showed a significant clinical and pathophysiological overlap with other syndromes involving connective tissues, only few cases of cerebrovascular events related to this syndrome have been described so far.
\end{abstract}

Case presentation: We report the case of a 33-years-old male diagnosed with ATS since childhood, that experienced three sudden episodes of expressive aphasia and right hemiparesis with spontaneous resolution. He was treated with recombinant tissue plasminogen activator ( $r$-TPA) at a dosage of $0.9 \mathrm{mg} / \mathrm{kg}$ with a complete recovery. Brain Magnetic Resonance Imaging (MRI) showed the absence of acute ischemic lesions and the patient was diagnosed with recurrent transient ischemic attacks (TIA).

Intracranial and supra-aortic trunks Magnetic Resonance Angiography (MRA) and Angio-CT scan of the thoracic and abdominal aorta showed marked vessel tortuosity without stenosis.

To our knowledge, this is the first reported case of an ATS patient with TIA in young age that was treated with intravenous thrombolysis with recombinant plasminogen activator.

Conclusion: Our report strengthens the relationship between ATS and juvenile cerebrovascular events, suggesting that an extensive study of body vessels in order to detect potential stenoses or occlusions in these cases is needed.

The greater predisposition to cerebrovascular events in ATS could benefit from a more aggressive primary and secondary prevention therapy.

Keywords: Arterial tortuosity syndrome, Transient ischemic attack, Thrombolysis

\section{Background}

Arterial Tortuosity Syndrome (ATS) is a rare, autosomal recessive connective tissue disorder characterized by elongated and tortuous large and medium-sized arteries

\footnotetext{
*Correspondence: stefanocottipiccinelli@gmail.com

${ }^{1}$ Neurology Unit, Department of Clinical and Experimental Sciences,

University of Brescia, Pz.le Spedali Civili 1, 25100 Brescia, Italy

Full list of author information is available at the end of the article
}

and a propensity to aneurysm formation, vascular dissection and stenosis of the pulmonary arteries.

ATS is caused by loss-of-function mutations in the SLC2A10 gene encoding the facilitative glucose transporter GLUT10 [1]. GLUT10 intracellular location remains uncertain and consequently also the exact pathogenesis of this syndrome. The main hypothesis is that SLC $2 A 10$ encodes a transporter of dehydroxyascorbic acid that may be present both over mitochondrial 
membranes and endoplasmic reticulum. Ascorbic acid is crucial for elastin and collagen maturation working as a hydroxylation cofactor for both prolyl and lysyl residues [2]. The reduction of hydroxylation of these residues appears to lead to impairment of vessel walls due to the perturbation of the transforming growth factor beta pathway caused by GLUT10 deficiency [3, 4]. Large vessel biopsies revealed reduction of smooth elastic lamella with highly disorganized, thicker and fragmented elastic fibers in vascular tissue [1].

Clinical onset usually occurs in infancy or early childhood. The most common manifestations are cardiovascular and include right ventricular hypertension, acute respiratory symptoms, ventricular hypertrophy and cardiac failure. Aneurysm formation and arterial dissection are frequent and may involve cerebrovascular circulation [1]. Other manifestations are dysmorphic features, keratoconus, hyperextensible skin, joint hypermobility, skeletal abnormalities, and generalized hypotonia [1].

Although ATS shows a significant clinical and pathophysiological overlap with other diseases involving connective tissues, only few cases of cerebrovascular events related to this syndrome have been described so far $[1,5,6]$.

Despite the description of several cases to date, clinical spectrum and natural history are still not completely understood and therefore, clinical management relies mainly on expert opinion [1].

\section{Case presentation}

We report the case of a 33-year-old Italian male born from consanguineous parents and diagnosed with ATS since adolescence carrying the homozygous c.1334delG (p.Gly445-Glufs"40) SLC2A10 pathogenic variant $[1,7]$.

$\mathrm{He}$ suffered a cardiac arrest at delivery. Reanimation was successfully performed, and no brain damages occurred. He had normal psycho-physical development. During childhood and adolescence his major alterations were tortuosity, dilatation, and elongation of the main arteries and an increase carotid bifurcation thickness of intima and media, associated with vomiting, failure to thrive, dyspnea, diaphoresis, arm and abdominal pain, and fainting. He also suffered recurrent fever, bronchitis, and pneumonia successfully treated with antibiotic therapy. At 19 years, aortic sinus dilation $(48 \mathrm{~mm})$ with a moderate aortic valve incompetence were detected by aortic angiography and computed tomography (CT). Doppler ultrasonography showed extreme narrowing and tortuosity of the arteries. Therefore, the patient underwent surgery by replacing the ascending aorta according to Tyrone-Davis procedure with Vascutek No.28 prosthesis and aortic valve sparing $[7,8]$. He also suffered from autoimmune chronic thyroiditis, treated with Levotiroxine $150 \mathrm{mcg}$ daily, and Gilbert' syndrome. He received cornea transplantation in left eye for corneal ectasia.

At 33 years, while he was at home, he experienced an episode characterized by expressive aphasia and right hemiparesis. Symptoms were described by family members lasting about $15 \mathrm{~min}$ with spontaneous resolution. While the patient was being transported to the hospital, he suffered a new episode with similar characteristics, lasting about $10 \mathrm{~min}$. A further episode was observed once the patients arrived at the emergency room. Neurological evaluation showed central deficit of the VII right cranial nerve, mild right hemiparesis, mild expressive aphasia with some naming deficit and mild dysarthria (National Institute of Health Stroke Scale, NIHSS =6). Brain CT showed no hyperacute signs of ischemia. Glucose, coagulation and blood cell counts were normal. After $20 \mathrm{~min}$, neurological picture began to improve spontaneously, but without
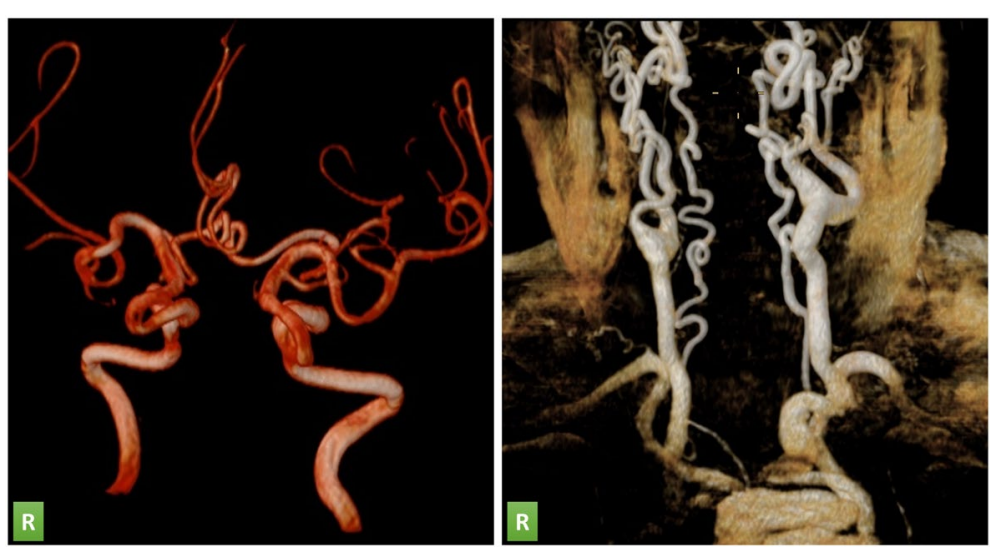

Fig. 1 Three-dimension reconstruction of Intracranial (on the left) and supra-aortic (on the right) trunks from Magnetic Resonance Angiography (MRA) of the ATS patient, showing a marked tortuosity of the vessels, in the absence of dissection, aneurysmal lesions or hemodynamically significant stenoses. R: right 


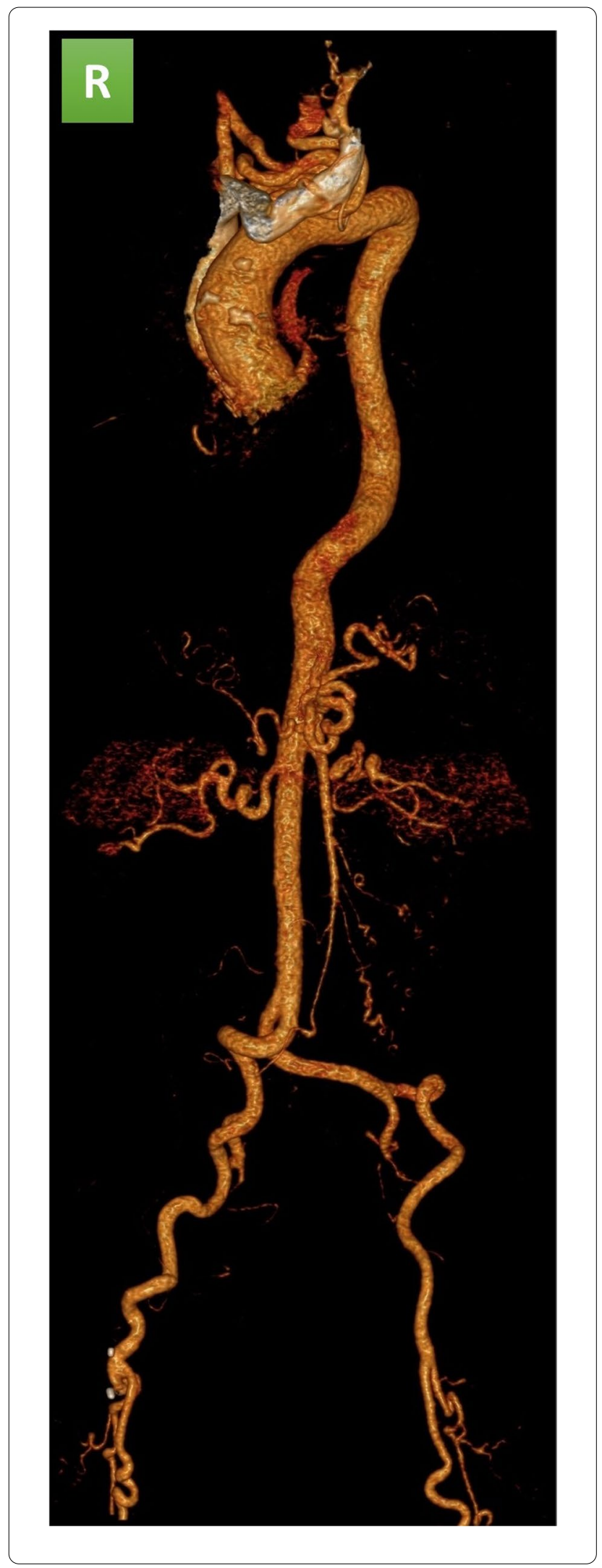

Fig. 2 Three-dimension reconstruction of Angio-CT scan of the thoracic and abdominal aorta of the ATS patient, showing tortuosity of thoracic and abdominal vessel without stenosis. R: right

a complete recovery. In accordance with the international stroke guidelines, the patient was admitted to the Stroke Unit and intravenous fibrinolytic therapy with recombinant tissue plasminogen activator (rTPA) at a dosage of $0.9 \mathrm{mg} / \mathrm{kg}$ was administered.

After $1 \mathrm{~h}$, the neurological examination was unremarkable. No signs of ischemia or bleeding complications were evident at brain CT after 24h. Electroencephalogram, urine analysis, electrolytes, coagulation and routine screening for juvenile stroke including thyroid ormones were normal.

3 Tesla brain magnetic resonance imaging (MRI) T1, T2 and FLAIR sequence showed the absence of acute ischemic lesions. Intracranial and supra-aortic trunks magnetic resonance angiography (MRA) showed a marked tortuosity of the vessels, in the absence of dissection, aneurysmal lesions or hemodynamically significant stenoses (Fig. 1)

Transesophageal echocardiography showed a moderate hypertrophy of the left ventricle with signs of diastolic dysfunction. There were no signs of patent foramen ovalis or shunt. Blood pressure values were monitored but were within normal limits.

Angio-CT scan of the thoracic and abdominal aorta showed tortuosity of thoracic and abdominal vessel without any stenosis (Fig. 2).

The patient was diagnosed with recurrent transient ischemic attacks (TIA) and discharged with Aspirin $100 \mathrm{mg}$ daily, with periodic follow-up and blood pressure monitoring indications.

At 3 months follow-up the patient was still asymptomatic.

\section{Discussion and conclusion}

Despite few cases published, our report helps to strengthen the relationship between ATS and juvenile cerebrovascular events. In a recent review of the published cases, Beyens et Al. reported two cases of juvenile stroke in ATS patients, one in association with intraparenchymal hemorrhage and one of unknown etiology resulting in left hemiparesis [1]. Cartwright et Al. reported a case of a 14 y.o female who presented with left basal ganglia ischemic stroke, but no others additional stroke risk factors were identified [5].

Since juvenile cerebrovascular events in ATS patients are rare, the mechanism by which ATS leads to cerebrovascular manifestations has not been established yet. Several hypotheses have been proposed. It may involve alterations of the endothelium and subsequent arterial 
thrombosis, vessel stenosis evolving to occlusion and infarction or dissection of affected arteries.

In this case, none of these anomalies were evident in intracranial vessels and supra-aortic trunks.

Since the patient carried a prosthesis of the ascending aorta, albeit with aortic valve sparing, an embolic genesis of the stroke cannot be excluded. However, imaging of the thoracic and abdominal vessels showed no significant abnormalities, except for the marked tortuosity of the vessels.

Thus, the origin of the recurrent transient ischemic attacks remains cryptogenic.

In consideration of the marked vessel alterations, patients suffering from this syndrome should be monitored closely for cerebrovascular risk factors and potential recurrences.

At diagnosis, imaging of cerebral and epiaortic vessels may be recommended in order to identify stenosis or aneurysms.

Furthermore, to our knowledge, this is the first reported case of an ATS patient treated with intravenous thrombolysis with rTPA. This treatment appeared to be effective and safe in the subject here described, supporting further future use in this type of patients.

The potential increased predisposition towards cerebrovascular events in ATS could suggest a benefit from an antiplatelet therapy, in these subjects, also as primary prevention strategy.

However, these preliminary findings could deserve further confirmations with studies on larger cohort of subjects with ATS.

\section{Patient perspective}

The patient and the patient's family were aware of the genetic diagnosis made years earlier but did not think it could generate any risk of cerebral ischemic events.

After hospitalization, the patient returned to a totally normal life and resumed work.

It was followed up by both clinical and telephone follow-up and, at the time of writing, no new episodes were presented.

The prospect that other similar events could occur worried the patient, but the patient was informed about what the early signs of cerebral ischemic events may be and about the need to be prompt in their recognition in order to obtain acute treatment.

\section{Abbreviations}

ATS: Arterial tortuosity syndrome; CT: Computed tomography; MRI: Magnetic resonance imaging; r-TPA: Recombinant tissue plasminogen activator.

\section{Acknowledgements}

Not applicable.

\section{Authors' contributions}

SCP, EP: drafted and revised the manuscript for intellectual content, followed patient in clinical setting. SB, NG, VV, MG, RS, ID, AC: followed patient in clinical setting, revised the manuscript. MR, RG: performed imaging and image processing procedures. AP, MM, MC, MB revised the manuscript for intellectual content. All authors have read and approved the manuscript.

\section{Funding}

No funding was obtained for this study.

Availability of data and materials

Data presented are available on request from the corresponding author.

\section{Declarations}

Ethics approval and consent to partecipate

Not applicable for ethics approval (no need of approval by ethics committee), written informed consent was obtained from the patient.

\section{Consent for publication}

Written informed consent was obtained from the patient for the publication of this case report and CARE guidelines were followed.

\section{Competing interests}

Authors declared no competing interests.

\section{Author details}

${ }^{1}$ Neurology Unit, Department of Clinical and Experimental Sciences, University of Brescia, Pz.le Spedali Civili 1, 25100 Brescia, Italy. ${ }^{2}$ Stroke Unit, Unità Operativa Neurologia Vascolare, ASST "Spedali Civili", Spedali Civili, Brescia, Italy. ${ }^{3}$ Martinos Center for Biomedical Imaging, Harvard Medical School, Massachusetts General Hospital, Charlestown, MA, USA. ${ }^{4}$ Radiology Unit, Department of Medical-Surgical Specialties, Radiological Sciences and Public Health, University of Brescia, Brescia, Italy. ${ }^{5}$ Division of Biology and Genetics, Department of Molecular and Translational Medicine, University of Brescia, Brescia, Italy. ${ }^{6}$ Neuroradiology Unit, Department of Medical-Surgical Specialties, Radiological Sciences and Public Health, University of Brescia, Brescia, Italy.

Received: 25 June 2021 Accepted: 20 October 2021

Published online: 30 November 2021

\section{References}

1. Coucke, P.J., Willaert, A., Wessels, M.W., Callewaert, B., Zoppi, N., De Backer, J., Fox, J.E., Mancini, G.M.S., Kambouris, M., Gardella, R., Facchetti, F., Willems, P.J., Forsyth, R., Dietz, H.C., Barlati, S., Colombi, M., Loeys, B., De Paepe, A., 2006. Mutations in the facilitative glucose transporter GLUT10 alter angiogenesis and cause arterial tortuosity syndrome. Nat Genet 38, 452-457. https://doi.org/https://doi.org/10. 1038/ng1764.

2. Beyens, A., Albuisson, J., Boel, A., Al-Essa, M., Al-Manea, W., Bonnet, D., Bostan, O., Boute, O., Busa, T., Canham, N., Cil, E., Coucke, P.J., Cousin, M.A., Dasouki, M., De Backer, J., De Paepe, A., De Schepper, S., De Silva, D., Devriendt, K., De Wandele, I., Deyle, D.R., Dietz, H., Dupuis-Girod, S., Fontenot, E., Fischer-Zirnsak, B., Gezdirici, A., Ghoumid, J., Giuliano, F., Diéz, N.B., Haider, M.Z., Hardin, J.S., Jeunemaitre, X., Klee, E.W., Kornak, U., Landecho, M.F., Legrand, A., Loeys, B., Lyonnet, S., Michael, H., Moceri, P., Mohammed, S., Muiño-Mosquera, L., Nampoothiri, S., Pichler, K., Prescott, K., Rajeb, A., Ramos-Arroyo, M., Rossi, M., Salih, M., Seidahmed, M.Z., Schaefer, E., Steichen-Gersdorf, E., Temel, S., Uysal, F., Vanhomwegen, M., Van Laer, L., Van Maldergem, L., Warner, D., Willaert, A., Collins, T.R., Taylor, A., Davis, E.C., Zarate, Y., Callewaert, B., 2018. Arterial tortuosity syndrome: 40 new families and literature review. Genet Med 20, 1236-1245. https:// doi.org/https://doi.org/10.1038/gim.2017.253.

3. Zaidi, S.H.E., Peltekova, V., Meyer, S., Lindinger, A., Paterson, A.D., Tsui, L.-C., Faiyaz-Ul-Haque, M., Teebi, A.S., 2005. A family exhibiting arterial tortuosity syndrome displays homozygosity for markers in the arterial tortuosity locus at chromosome 20q13. Clin Genet 67, 183-188. https:// doi.org/https://doi.org/10.1111/j.1399-0004.2004.00391.x. 
4. Zoppi, N., Chiarelli, N., Cinquina, V., Ritelli, M., Colombi, M., 2015. GLUT10 deficiency leads to oxidative stress and non-canonical av $\beta 3$ integrinmediated TGF $\beta$ signalling associated with extracellular matrix disarray in arterial tortuosity syndrome skin fibroblasts. Hum Mol Genet 24, 6769-6787. https://doi.org/https://doi.org/10.1093/hmg/ddv382.

5. Cartwright, M.S., Hickling, W.H., Roach, E.S., 2006. Ischemic stroke in an adolescent with arterial tortuosity syndrome. Neurology 67, 360-361. https://doi.org/https://doi.org/10.1212/01.wnl.0000225056.66762.f9.

6. Wei F; Diedrich K.T, Fullerton H.J., deVeber G, Wintermark M, Hodge J, Kirton A., and the Vascular Effects of Infection in Pediatric StrokeArterial Tortuosity: An imaging biomarker of childhood stroke pathogenesis? Stroke. 2016 May; 47(5): 1265-1270.

7. Gardella, R., Zoppi, N., Assanelli, D., Muiesan, M.L., Barlati, S., Colombi, M., 2004. Exclusion of candidate genes in a family with arterial tortuosity syndrome. Am J Med Genet A 126A, 221-228. https://doi.org/https://doi. org/10.1002/ajrmg.a.20589.

8. Bottio, T., Bisleri, G., Piccoli, P., Muneretto, C., 2007. Valve-sparing aortic root replacement in a patient with a rare connective tissue disorder: arterial tortuosity syndrome. J Thorac Cardiovasc Surg 133, 252-253.https:// doi.org/https://doi.org/10.1016/j.jtcvs.2006.08.050.

\section{Publisher's Note}

Springer Nature remains neutral with regard to jurisdictional claims in published maps and institutional affiliations.

- fast, convenient online submission

- thorough peer review by experienced researchers in your field

- rapid publication on acceptance

- support for research data, including large and complex data types

- gold Open Access which fosters wider collaboration and increased citations

- maximum visibility for your research: over 100M website views per year

At BMC, research is always in progress.

Learn more biomedcentral.com/submissions 